\title{
Accréditation des cadres de premier niveau : a-t-on ouvert une boîte de Pandore?
}

\section{Daniel Gagnon $^{\text {a }}$}

RÉSUMÉ. En fin d'année 2016, après un va-et-vient devant différentes instances judiciaires débuté en 2009, le Tribunal administratif du travail (TAT) décrétait que l'exclusion des cadres du droit d'association prévue au Code du travail ne s'appliquait pas pour les demandes déposées par les cadres de premier niveau chez Hydro-Québec ainsi qu'à la Société des casinos du Québec. Au terme de cette décision, doit-on conclure qu'à l'avenir tous les cadres de premier niveau auront droit à l'accréditation syndicale? Si tel est le cas, quelles seront les conséquences sur les unités de négociation existantes et, de façon plus générale, sur les relations de travail chez les employeurs visés par ces nouvelles demandes d'accréditation? Dans le présent exposé, nous ferons état des démarches entreprises devant les instances judiciaires pour nous pencher par la suite sur les motifs ayant justifié la décision du TAT, le tout à la lumière de l'évolution du droit d'association tel qu'établi par la Cour suprême du Canada. Au terme de cette analyse, le lecteur devrait être en mesure d'avoir sa propre opinion quant à l'issue potentielle des demandes d'accréditation déposées par des cadres de premier niveau.

ABSTRACT. At the end of 2016, after a back and forth in front of different judicial bodies started in 2009, the Administrative Labor Tribunal (TAT) decreed that the exclusion of the right of association provided in the Quebec Labor Code did not apply to Hydro-Québec and the Societé des casinos for their first level managers. At the end, should we conclude that in a near future all first level managers will be entitled to union accreditation? If that is the case, what will be the consequences for existing bargaining units and more generally on the labor relations of the employers targeted by this news applications for unionization? In this presentation, we will discuss the steps taken in judicial proceedings to consider later the reasons that justified the decision of the TAT, all in the light of the evolution of the right of association as established by the Supreme Court of Canada. At the end of this analysis, the reader should be able to have his or her own opinion as to the potential outcome of the requests accreditation filed by first level managers.

\section{Introduction}

Le 7 décembre 2016, la juge Irène Zaikoff du Tribunal administratif du travail de Québec rendait deux décisions ${ }^{1}$ qui risquent de bouleverser l'ordre établi dans les relations de travail au Québec. Bien que le Code du travail (RLRQ, c. C -27) ne reconnaisse pas le statut de salarié aux personnes cadres en entreprise, l'«Association des cadres de la Société des casinos du Québec » tout comme l'« Association professionnelle des cadres de premier niveau d'Hydro-Québec » ont obtenu gain de cause dans un recours à l'issue duquel la disposition excluant le personnel cadre du droit d'association (prévue au sous-paragraphe 1 l) $1^{\circ} \mathrm{du}$
Code du travail) fut déclarée inapplicable à ces deux associations. Dans la foulée de la position prise par la Cour suprême du Canada au cours des dernières années, la juge Zaikoff statua que le sousparagraphe $1 /) 1^{\circ}$ portait atteinte au droit à l'exercice de la liberté d'association, liberté protégée à l'alinéa 2d) de la Charte canadienne des droits et libertés (partie I de la Loi constitutionnelle de 1982 sur le Canada; 1982, c. 11 (R.-U.)) tout comme à l'article 3 de la Charte des droits et libertés de la personne (RLRQ, c. C -12).

Étoffé, ce jugement effectue une rétrospective du droit à l'association pour certaines personnes agissant à titre de représentants de l'employeur. Toutefois,

${ }^{a}$ Professeur, avocat, CRHA, Université du Québec à Chicoutimi 
cette décision concernant des cadres de premier niveau doit être circonstanciée, d'où la réserve émise par plusieurs juristes, tout comme par la juge Zaikoff elle-même, d'éviter de conclure de façon générale à l'inconstitutionnalité automatique et, ce faisant, l'inclusion de tout personnel cadre à la définition de salarié prévue au Code du travail. Les deux cas à l'étude mettent en cause des sociétés d'État comprenant au moins cinq paliers de gestion; se pose alors la question de savoir s'il en irait autrement chez un employeur du secteur privé ou encore dans un milieu de travail où la structure hiérarchique serait de moindre envergure?

Nonobstant cette réserve, y a-t-il lieu de croire que la décision de la juge Zaikoff, dans un futur rapproché, servira d'assise à d'autres demandes d'accréditation de cadres au sein d'organisations de travail comportant plusieurs niveaux hiérarchiques? Si tel est le cas, quelles seront les conséquences à moyen terme pour la gestion des relations de travail au sein de ces entreprises?

\section{Chronologie des procédures soumises aux tribunaux}

\subsection{La requête en accréditation de l'Association des cadres de la Société des casinos du Québec (novembre 2009)}

Le 10 novembre 2009, l'Association des cadres de la Société des casinos du Québec dépose auprès de la Commission des relations du travail (CRT) ${ }^{2}$ une requête en accréditation conformément aux dispositions du Code du travail pour représenter :

Les cadres de premier niveau (classes 4 et $4 \mathrm{a}$ ) du secteur des jeux, notamment ceux offerts aux tables de jeux, aux machines à sous/keno, aux salons de poker et à tout autre système de loterie de même nature aux fins de l'exploitation d'un casino d'État, et ce pour l'établissement de l'employeur situé au 1, avenue du Casino, Montréal (Québec).

Dans le contexte de cette requête, l'Association des cadres demande à la CRT en vue de procéder à l'accréditation de l'unité de négociation visée de :

Déclarer que l'exclusion du statut de cadre prévue à l'article 1 l) paragraphe $1 \mathrm{du}$ Code du travail lui est inopposable constitutionnellement puisqu'elle porte directement atteinte à l'exercice de la liberté d'association par l'association requérante et, particulièrement, porte atteinte à la liberté d'association des employés faisant partie du groupe visé par la présente requête en accréditation.

Le 23 novembre 2009, l'association requérante précisait sa prétention en ajoutant l'argumentation suivante au libellé de sa demande d'accréditation :

[...] laquelle liberté, selon les plus récentes décisions des tribunaux canadiens et québécois comprend le droit d'association, le droit à la reconnaissance par l'employeur d'une association d'employés et le droit à la négociation d'une entente collective de travail.

Le lendemain, 24 novembre 2009, une requête en irrecevabilité fut déposée par la Société des casinos du Québec où il était allégué que la CRT n'avait pas compétence pour se prononcer sur la question constitutionnelle que soulevait la demande d'accréditation. Selon les procureurs de l'employeur, une des parties, en l'occurrence l'association des cadres, n'est pas couverte par les dispositions du Code du travail, le personnel cadre étant nommément exclu de la définition de "salarié » selon le sous-paragraphe 1 l) $1^{\circ}$ du Code $^{3}$. Ce faisant, la CRT ne serait pas valablement saisie du litige et ne pourrait donc décider des questions constitutionnelles qui lui sont soumises.

Dans une décision du 14 avril 2010, la CRT rejette l'argument de l'employeur et se déclare compétente pour entendre l'affaire ${ }^{4}$. La Cour supérieure annulera par la suite cette décision le 23 janvier 2012 au terme d'une requête en révision judiciaire déposée par l'employeur ${ }^{5}$. Selon la Cour supérieure, la CRT ne pouvait trancher la question de la constitutionnalité d'une disposition du Code du travail, cet exercice devenant l'apanage unique de la Cour supérieure. Ainsi, la CRT ne pouvait valablement être saisie du litige puisque les personnes visées par la requête en accréditation ne sont pas couvertes par les dispositions du Code du travail. Le juge Reimnitz y allait du commentaire suivant qui résume assez bien la position justifiant sa décision :

(36) Il y a dans ce dossier, des considérations particulières qui font en sorte que la question en cause est tellement importante qu'elle se doit d'être adressée à la Cour supérieure. Il 
semble tout à fait raisonnable de ne pas laisser à un tribunal administratif le soin de décider d'appliquer à un nouveau groupe de travailleurs, soit les cadres de la Société des casinos du Québec, le régime particulier prévu par le Code du travail pour les salariés. Surtout qu'il a toujours été reconnu et accepté que l'Association des cadres ne répond pas à la définition de «salarié » au sens de $1 \mathrm{l}$ ) du Code du travail. [...]

(43) N'ayant pas de compétence pour se prononcer sur des questions de droit touchant les cadres, demander à la CRT de se prononcer sur une question constitutionnelle comme celle en cause, revient à lui demander de se prononcer de façon déclaratoire, ce qu'elle n'a pas le pouvoir de faire. Cette question doit être soumise au tribunal de droit commun soit la Cour supérieure ${ }^{6}$.

Conséquemment, le juge Reimnitz accueillait la requête en révision judiciaire et rejetait la requête en accréditation déposée par l'Association des cadres de la Société des casinos du Québec.

Le 24 mars 2014, la Cour d'appel du Québec, dans un jugement unanime, infirma cette décision. Bien que le plus haut tribunal de la province prenne soin de préciser que l'association requérante reconnaissait d'emblée que les personnes visées par la requête en accréditation sont des cadres 7 , il y avait lieu selon la Cour d'appel de distinguer en la présente affaire.

Le recours intenté dans le présent cas vise à faire déclarer inconstitutionnelle la disposition du Code du travail sur la base de laquelle la demande d'accréditation avait été refusée, argument qui n'a pas été soumis antérieurement à la Cour d'appel et sur lequel, en conséquence, aucun tribunal n'a statué à ce jour. S'appuyant sur la décision Conway ${ }^{8}$ de la Cour suprême, la Cour d'appel cite le passage suivant de ladite décision, lequel est très explicite au regard du point de droit soumis :

(77) Ces arrêts confirment que le tribunal administratif investi du pouvoir de trancher des questions de droit et dont la compétence pour appliquer la Charte n'est pas clairement écartée a le pouvoir - et le devoir - correspondant d'examiner et d'appliquer la Constitution, y compris la Charte, pour se prononcer sur ces questions de nature juridique $[\ldots]^{9}$.
Selon le plus haut tribunal de la province, la CRT a le pouvoir de trancher des questions de droit en vertu de l'article 118 du Code du travail ${ }^{10}$ et sa compétence en matière constitutionnelle n'a pas été écartée par le législateur. La requête en accréditation ayant été déposée conformément aux dispositions du Code $d u$ travail, la CRT en est valablement saisie, ce qui lui confère en vertu des dispositions du Code du travail une juridiction exclusive sur celle-ci.

Au terme de ce jugement, le dossier retourne à la CRT afin que cette dernière statue sur la requête en accréditation. Pour ce faire, elle devra inévitablement trancher la question constitutionnelle soumise. Ainsi, à la suite d'une conférence préparatoire tenue en septembre 2014 avec les parties, la Commission des relations du travail (CRT) formulait la question constitutionnelle à débattre de la façon suivante :

1- Cette exclusion porte-t-elle atteinte à la liberté d'association garantie par l'article $2 \mathrm{~d}$ ) de la Charte canadienne des droits et libertés et par l'article 3 de la Charte des droits et libertés de la personne des personnes visées par la requête en accréditation?

2- Dans l'affirmative, cette atteinte est-elle justifiée en regard de l'article 1 de la Charte canadienne et de l'article 9.1 de la Charte québécoise ${ }^{11}$ ?

\subsection{La plainte pour modification unilatérale des conditions de travail de l'Association des cadres de la Société des casinos du Québec (avril 2012)}

Le 23 avril 2012, l'Association de cadres, au moyen d'une procédure de grief préventif, demanda que soit déféré à l'arbitrage le fait que, selon elle, l'employeur modifiait de façon illégale depuis mars 2012 les conditions de travail en réduisant considérablement les heures de travail (articles 59 et 100.10 CT) ${ }^{12}$.

Le 13 juin 2012, la Société des casinos du Québec conteste la compétence de l'arbitre en cette affaire pour les motifs constitutionnels précédemment exposés et le 7 septembre suivant, le tribunal d'arbitrage rejette cette contestation. La décision prend ses assises sur celle de la CRT rendue le 14 avril 2010 où la CRT décidait qu'elle avait compétence pour traiter la 
demande en accréditation. Pour l'arbitre, la CRT devant ultérieurement statuer sur la présomption de constitutionnalité de la loi, lui n'a pas à se soucier de cet argument à ce stade-ci des procédures.

Une requête en révision judiciaire de la décision de l'arbitre s'ensuivit au terme de laquelle la Cour supérieure détermina le 10 septembre 2015 que l'arbitre de grief avait compétence pour procéder sur le fond de la plainte de l'article 59, CT et ce, avant même que le litige constitutionnel ne connaisse sa conclusion ${ }^{13}$. Le passage suivant résume assez bien le fondement juridique de la décision du juge Labelle :

(11) Par sa décision, l'Arbitre ne statue pas sur le fond du litige dont il est saisi soit le maintien ou non par l'Employeur des conditions de travail des membres de l'Association et le droit de ces derniers à l'obtention d'une ordonnance de sauvegarde à cet effet.

(12) En disposant de la requête en irrecevabilité de l'Employeur, l'Arbitre affirme sa compétence à entendre la Plainte et à trancher son bien-fondé.

(13) La jurisprudence est à l'effet que, sauf si des circonstances exceptionnelles le justifient, il faut laisser l'Arbitre exercer sa juridiction jusqu'au bout du processus de la plainte. Une requête en irrecevabilité ne sera accordée que de façon exceptionnelle ${ }^{14}$.

Cette décision du juge Pierre Labelle fit l'objet d'une demande d'autorisation à la Cour d'appel du Québec. La juge Marie-France Bich autorisa le recours le 19 novembre 201515. Le passage suivant de sa décision mérite qu'on s'y attarde :

(4) L'affaire présente en effet un caractère exceptionnel. Certes, elle paraît de prime abord se heurter à l'écueil du caractère interlocutoire de la sentence arbitrale, mais, malgré cela, l'affaire soulève une intéressante question de principe, nouvelle de surcroît, qu'il paraît opportun de soumettre à la Cour ${ }^{16}$.

La Cour d'appel se prononça par la suite le

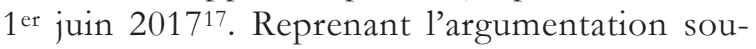
mise aux instances précédentes, la Société des casinos du Québec reprochait à ces dernières d'avoir statué sans égard à la présomption de constitutionnalité des dispositions législatives (le sous-paragraphe 1 l) $1^{\circ} \mathrm{du}$ Code du travail) dont la validité doit être tenue pour acquise jusqu'à décision définitive à l'effet contraire.

Dans une décision unanime, encore sous la plume de la juge Bich, la Cour d'appel rejette cette prétention, commentant au passage la prématurité de la demande déposée par la Société des casinos du Québec :

(28) Cela dit, plutôt que de rejeter la demande de contrôle judiciaire de l'appelante au motif de sa prématurité, le juge l'a tranchée, concluant à la compétence de l'arbitre sur la mésentente. Ce faisant, a-t-il erré?

(29) Dans les circonstances, je réponds à cette question par la négative et je suggère d'ailleurs que la Cour, à l'instar du juge d'instance, statue elle-même sur le fond du moyen préliminaire que soulève l'appelante. Pourquoi? C'est qu'à ce stade de l'affaire, renvoyer celle-ci à l'arbitre sans trancher serait une perte de temps de nature à engendrer une grave injustice pour l'une et l'autre [des] parties. [...] Retourner le dossier à l'arbitre sans résoudre la question risquerait simplement de provoquer, au moment de la sentence arbitrale finale, une répétition de l'actuel débat et de retarder encore les choses. Il paraît donc, par exception, nécessaire de décider du moyen préliminaire soulevé par l'appelante ou, plus exactement, de vérifier si le juge de première instance a commis une erreur en se prononçant comme il l'a fait ${ }^{18}$.

Par la suite, la Cour d'appel traduit très bien l'essence du droit applicable (tel que l'avait perçu l'arbitre de grief Malette) en refusant d'intervenir :

(42) En somme, selon l'arbitre, la protection offerte par l'art. 59 C.t. et le droit d'user du recours prévu par l'article 100.10 C.t. découlent du simple fait qu'une requête en accréditation a été déposée auprès de l'instance compétente en cette matière (CRT à l'époque, TAT aujourd'hui). Dès ce moment, l'employeur ne peut plus modifier les conditions de travail des personnes visées par la requête, et ce, peu importe que la recevabilité de celle-ci ou son bien-fondé ou 
le statut des personnes qu'elle vise soit contesté devant la CRT ou le TAT, instance qui seule peut statuer là-dessus. L'employeur se trouve ainsi empêché d'exercer une pression sur les intéressés en améliorant ou, au contraire, en érodant leurs conditions de travail. $[\ldots]$

(49) D'autre part, une fois la requête en accréditation déposée auprès de la CRT (ou du TAT), c'est à l'arbitre désigné en vertu de l'article 100.10 C.t. de voir à l'application de l'art 59 C.t. (dont le premier alinéa est en cause ici). Quelle est l'ampleur du mandat que lui attribuent ces dispositions? Il est raisonnable de penser, comme l'écrit l'arbitre Malette, que tout ce qui concerne la requête en accréditation comme telle, qu'il s'agisse de fond, de forme ou de recevabilité ne le regarde pas et ne ressortit pas à sa compétence. C'est là une conclusion respectueuse de la compétence exclusive de la CRT (ou du TAT), mais aussi de l'économie d'un Code qui, de façon générale, ne favorise pas la concurrence juridictionnelle.

$[\ldots]$

(57) Conciliant les champs de compétence respectifs de l'arbitre saisi d'une mésentente en vertu des art. 59 et 100.10 C.t. et du TAT (CRT) saisi d'une requête en accréditation, on doit conclure que ce n'est pas au premier de décider si les personnes au nom desquels on réclame l'application de cette disposition sont ou non des salariés au sens du paragr. 1l) C.t., mission qui incombe exclusivement au TAT (précédemment CRT). Cela étant, il faut interpréter en conséquence l'art. 59 et voir que les " salariés » au bénéfice desquels cette disposition est édictée sont plutôt « les personnes visées par la requête en accréditation ", peu importe leur statut juridique réel, qui sera décidé par le TAT $[\ldots]^{19}$.

\subsection{La requête en accréditation de l'Association professionnelle des cadres de premier niveau d'Hydro-Québec (décembre 2014)}

Le 19 décembre 2014, l'Association professionnelle des cadres de premier niveau d'Hydro-Québec dépose une requête en accréditation libellée comme suit :
Tous les cadres de premier niveau à l'emploi d'Hydro-Québec à l'exclusion des cadres avec relevants cadres, des cadres assujettis au SPIHQ, des cadres de la fonction Ressources humaines, des cadres régis par le Répertoire des conditions de travail chantier (RCTC), des cadres occupant des fonctions de nature stratégique ou confidentielle et des cadres affectés à des projets spéciaux.

\subsection{La requête en réunion de dossiers déposés par l'Association professionnelle des cadres de premier niveau d'Hydro-Québec (janvier 2015)}

Le 8 janvier 2015, l'Association professionnelle des cadres de premier niveau d'Hydro-Québec demande à la CRT de réunir son dossier avec celui de l'Association des cadres de la Société des casinos du Québec, les points de droit soumis étant, selon sa prétention, similaires.

Nonobstant le fait que la trame factuelle varie dans les deux environnements de travail, la CRT accueille la requête le 30 janvier 2015 et réunit les deux dossiers.

\subsection{La plainte pour entrave à l'activité associative de l'Association des cadres de la Société des casinos du Québec (février 2015)}

Le 26 février 2015, une plainte fut déposée par l'Association des cadres de la Société des casinos du Québec à la suite du licenciement de 10 de ses membres le 28 janvier 2015. L'association allègue qu'il y a là contravention à l'article 12 du Code $d u$ travail $^{20}$ au motif que cela constitue une entrave à ses activités associatives.

Le lendemain, 27 février, la Société des casinos du Québec soulevait une objection préliminaire sur la base que l'association ne représente que du personnel cadre. Ainsi, le recours de l'article 12 CT étant réservé à une association de "salariés ", celui-ci n’aurait donc pas lieu d'être, d'autant plus que la présomption de constitutionnalité de la loi rend irrecevable la plainte tant qu'un tribunal n'aura pas décrété l'inconstitutionnalité du sous-paragraphe 1 l) de l'article 1. 
Toute loi devant être considérée comme valide, les tribunaux ne peuvent présumer de leur inconstitutionnalité21. C'est sur la base de ce principe établi par la Cour d'appel du Québec que, le 25 juin 2015, la CRT rejetait la requête en irrecevabilité de la Société des casinos du Québec et suspendait la procédure intentée en vertu de l'article 12 du Code du travail par l'Association des cadres de la Société des casinos du Québec jusqu'au prononcé de la décision finale sur la requête en accréditation :

[40] [...] La requête en irrecevabilité est rejetée parce qu'il serait prématuré de mettre fin au litige avant même que la Commission n'ait eu l'opportunité de trancher la question constitutionnelle relative à la légalité de l'exclusion des cadres de la notion de "salariés » prévue au sous-paragraphe 1 du paragraphe l) de l'article $1 \mathrm{du}$ Code $^{22}$.

Ainsi donc, au terme de cet aller-retour des parties devant les tribunaux (sur une période de cinq ans et demi), la table était mise en 2016 pour que le Tribunal administratif du travail tranche enfin le débat de fond : les cadres de premier niveau des deux associations requérantes sont-ils exclus de la notion de salariés du Code du travail et, advenant une réponse négative, peuvent-ils former une association de salariés conformément au Code du travail en vue de négocier collectivement leurs conditions de travail?

\section{La décision du Tribunal administratif du travail sur les deux requêtes en accréditation}

Le 29 juillet 2016, la juge administrative Zaikoff prenait en délibéré l'argumentation soumise par les parties en vue de rendre sa décision sur les deux dossiers le 7 décembre suivant. L'ampleur du texte (102 pages dans un cas et 94 dans l'autre) explique en soi le délai23. Dès le premier paragraphe, elle situe les paramètres de la décision à intervenir :

[1] La présente décision, ainsi que celle qui est rendue ce même jour dans le dossier CM 2009-5820, porte sur la constitutionnalité, en regard du droit à la liberté d'association, de l'exclusion du statut de cadre de la définition de «salarié » prévue au Code du travail (le Code), condition nécessaire à l'accréditation d'une association aux fins de la négociation collective ${ }^{24}$.
Comme énoncé précédemment, une conférence préparatoire entre les parties impliquées avait circonscrit le débat constitutionnel aux deux questions suivantes : l'exclusion porte-t-elle atteinte à la liberté d'association et, dans l'affirmative, cette atteinte est-elle acceptable dans ce que les tribunaux conviennent d'appeler " une société libre et démocratique 25 » nonobstant l'atteinte à un droit garanti par les Chartes?

\subsection{Historique de la syndicalisation des cadres au Québec et autres éléments pertinents}

Des témoins experts tout comme d'autres venus relater les éléments factuels des deux cas à l'étude ont été entendus en cours d'audience. Dans un premier temps, les dispositions de certaines conventions internationales portant sur les droits fondamentaux du travail ont été analysées ${ }^{26}$. Par la suite, le tribunal a précisé la portée du terme " cadre », départageant en trois niveaux les personnes assignées à la direction d'une organisation. L'expression « cadre supérieur » vise des personnes investies de fonctions qui relèvent directement du conseil d'administration ou de la haute administration de l'entreprise. Le « cadre intermédiaire » sera celui impliqué au niveau de l'élaboration des politiques de l'entreprise tandis que le « cadre de premier niveau » s'assure que les directives de l'employeur sont respectées par les salariés.

La partie suivante de la décision porte sur ce qu'il en est au niveau de la législation fédérale du droit du travail, faisant état du fait que le Code canadien du travail (L.R.C. (1985) ch. L-2) prévoit à la définition du terme " employé » une exclusion pour les personnes qui n'occupent que des fonctions de direction ${ }^{27}$. Du coup, des "employés » au sens de ce texte de loi peuvent être des personnes qui ont uniquement des fonctions de surveillance (et non de direction). Voilà pourquoi, au fil des ans, les réalisateurs de la Société Radio-Canada, les surintendants d'une entreprise de débardage tout comme les capitaines de navires et certains superviseurs d'entreprises de téléphonie ont pu être accrédités selon le Code canadien du travail28. En somme, des personnes à qui l'on peut attribuer le qualificatif de « cadres de premier niveau »!

Un exposé prenant ses assises sur le rapport du professeur Michel Coutu de l'École des relations industrielles de l'Université de Montréal est venu préciser l'évolution historique du sujet. Dans la 
province de Québec, dès les débuts de la législation en matière de contrat de travail collectif, les cadres (de quelque niveau que ce soit) ont été exclus de la notion de salarié. Ainsi, il ne leur était pas possible de faire partie d'une association accréditée lors de l'adoption de la Loi des relations ouvrières en 194429.

L'exclusion des cadres du processus de syndicalisation se poursuivit avec l'adoption du Code du travail en 1964. À partir de là, toutefois, il fut permis aux professionnels de former une association accréditée. Deux syndicats voient ainsi le jour : le «Syndicat professionnel des ingénieurs de la Ville de Montréal et de la Communauté urbaine de Montréal » (en 1963) et le "Syndicat professionnel des ingénieurs d'Hydro-Québec » (en 1964). Notons que dans les deux cas, les ingénieurs cadres sont inclus dans l'unité de négociation existante faisant en sorte qu'il ne s'agit pas à proprement parler d'associations accréditées officiellement selon le Code du travail mais plutôt d'associations volontairement reconnues par l'employeur. Dans la même lignée, la "Fédération des ingénieurs cadres du Québec» (devenue par la suite la «Fédération des professionnels salariés et cadres du Québec »), affiliée à la CSN, existera jusqu'à sa dissolution en 1979.

En 1969, le Code du travail est amendé et la reconnaissance volontaire d'association est alors abolie. Un amendement apporté l'année suivante à la législation permettra aux associations déjà reconnues chez Hydro-Québec et à la Ville de Montréal de survivre. Il en sera de même pour l'« Association des contremaîtres » à la Ville de Montréal ${ }^{30}$.

En 1978, le gouvernement provincial reconnaît par décret l'« Association des cadres supérieurs » aux fins des relations de travail. Plusieurs autres décrets reconnaissant des associations de cadres à la fonction publique suivront. À partir des années 1990, des associations de cadres seront regroupées en confédération et fédérations.

Nonobstant l'adoption de ces décrets gouvernementaux, la reconnaissance officielle des associations de cadres par voie législative est exceptionnelle et se limite aux deux employeurs ayant reconnu antérieurement à 1969 des associations de cadres, soit la Ville de Montréal et Hydro-Québec.

Au-delà de ces deux groupes, des associations de cadres du secteur public des établissements scolaires ou de la santé et des services sociaux seront également établies, mais encore là par décrets gouvernementaux.

Le professeur Coutu poursuit son témoignage en précisant qu'il existe d'autres associations de cadres reconnues par entente avec l'employeur, entre autres, dans le secteur universitaire tout comme chez certaines sociétés d'État. Toutefois, seules des associations « accréditées » ont accès à un véritable processus de négociation collective. Certaines associations ont des échanges avec leur employeur, mais celui-ci décide seul en cas d'impasse. Dans d'autres cas, une consultation préalable est effectuée conformément à l'entente de travail, mais la détermination ou la modification des conditions de travail résulte d'une décision unilatérale de l'employeur et non pas d'un processus de négociation.

Les cas d'associations de cadres chez les employeurs du secteur dit privé sont pratiquement inexistants aux dires du professeur Coutu.

Bien que le tribunal n'adhère pas à la totalité des éléments contenus au rapport du professeur Coutu, la juge Zaikoff précise tout de même que ce rapport «demeure utile et probant dans son ensemble ${ }^{31}$.

\subsection{Les plaintes d'associations de cadres provinciales au niveau international}

Le 18 mars 2003, la Confédération nationale des cadres du Québec (CNCQ) ${ }^{32}$ et trois associations de cadres de sociétés d'État, soit l'Association des cadres de la Société des casinos du Québec (ACSCQ), l'Association professionnelle des cadres de premier niveau d'Hydro-Québec (APCPNHQ) et l'Association des directeurs et directrices de succursale de la SAQ (ADDS/SAQ), déposent une plainte au Comité sur la liberté syndicale de l'Organisation internationale du travail (OIT). En novembre 2004, le Comité sur la liberté syndicale conclut que l'exclusion des cadres du régime général du Code $d u$ travail ne respecte pas les engagements internationaux du Canada en la matière, plus spécifiquement la Convention 87 et la Convention 98 :

463. Au vu de tous ces éléments, le comité demande au gouvernement d'amender les dispositions pertinentes du Code du travail du Québec afin que les cadres jouissent du droit de bénéficier du régime général de droit de travail collectif et de constituer des associations jouissant des 
mêmes droits, prérogatives et voies de recours que les autres associations de «salariés $»^{33}$.

Au terme de ce constat, le comité formulait trois recommandations, dont la suivante :

470. Au vu des conclusions qui précèdent, le comité invite le conseil d'administration à approuver les recommandations suivantes :

a) Le comité demande au gouvernement d'amender le Code du travail du Québec afin que les cadres aient le droit de bénéficier du régime général de droit du travail collectif et de constituer des organisations jouissant des mêmes droits, prérogatives et voies de recours que les autres organisations de travailleurs, notamment en ce qui concerne les mécanismes de négociation collective et de règlement des différends et la protection contre les actes de domination et d'ingérence des employeurs, le tout conformément aux principes de la liberté syndicale ${ }^{34}$.

À la suite d'un suivi de la mise en œuvre de ses recommandations, le Comité sur la liberté syndicale de l'OIT déplorera l'absence de progrès et requerra des explications de la part du gouvernement provincial. Il le fera à plusieurs reprises subséquemment sans que la situation évolue. Le dépôt de la requête en accréditation devant la Commission des relations du travail (CRT) en novembre 2009 amène le gouvernement du Québec à réserver ses commentaires auprès du Comité sur la liberté syndicale de l'OIT. Dans un rapport de suivi de novembre 2011, le comité rapporte les observations suivantes du gouvernement du Québec :

Le Procureur général du Québec, agissant pour et au nom du gouvernement du Québec, est également partie à ces procédures judiciaires, lesquelles sont toujours en cours. Le gouvernement ajoute que les parties maintiennent ainsi une certaine forme de dialogue, qui n'exclut pas que des négociations, des consultations ou des échanges d'informations puissent avoir lieu entre les représentants des parties ${ }^{35}$.

Malgré une demande réitérée en octobre 2013 par le Comité sur la liberté syndicale de l'OIT pour une modification législative du Code du travail (afin de le rendre conforme aux principes de la liberté syndicale), la juge Zaikoff constate que la situation n'a pas changé à ce jour.

\subsection{Les particularités de la preuve propres à chaque dossier}

\subsubsection{Chez Hydro-Québec}

Hydro-Québec se décline en six paliers hiérarchiques de gestionnaires : le président-directeur général nommé par le conseil d'administration, le président, les vice-présidents, les directeurs, les cadres intermédiaires et les cadres de maîtrise, appelés aussi cadres de premier niveau.

Les cadres de premier niveau sont en partie ceux visés par la requête en accréditation. Au nombre de 800 , ils sont répartis sur tout le territoire couvert par la société d'État.

Hydro-Québec gère environ 20000 employés dont la majorité, syndiquée, est répartie au sein de neuf unités de négociation. Parmi les associations de cadres que compte la société d'État se retrouve le Syndicat professionnel des ingénieurs d'HydroQuébec (SPIHQ), dont certains de ses membres sont cadres de premier niveau et supervisent des salariés syndiqués. Ils font partie de la même unité de négociation que ces derniers, laquelle est régie par une convention collective conclue en vertu des dispositions du Code du travail.

L'Association professionnelle des cadres de premier niveau d'Hydro-Québec (APCPNHQ) fut créée en 1985 en vue principalement d'améliorer les conditions de travail de ses membres et leur sécurité d'emploi. Elle compte 700 membres.

Au terme de plusieurs rencontres entre les parties, Hydro-Québec s'est montrée ouverte à reconnaître l'APCPNHQ, mais a voulu éviter tout vocable qui laisserait croire à l'existence d'un syndicat et à une négociation collective. C'est dans ce contexte que les parties ont signé une première entente le 11 juin 1986 où l'article 2 du document stipule :

2. Reconnaissance de l'Association

L'entreprise, dans son esprit de collaboration et de concertation, reconnait l'Association comme : 
a) le seul organisme représentatif et, par conséquent, habilité à discuter, rechercher et convenir avec l'entreprise d'ententes relatives aux conditions de travail des cadres de premier niveau;

b) le seul organisme représentatif habilité à représenter les cadres de premier niveau membres de l'Association auprès d'Hydro-Québec sur l'application de leurs conditions de travail ${ }^{36}$.

Plus tard dans l'année, l'association sera constituée en vertu des dispositions de la Loi sur les syndicats professionnels (RLRQ c. S -40) et, conformément aux dispositions de l'article 6 de cette loi, ses statuts et règlements précisent qu'elle vise l'étude, la défense et le développement des intérêts économiques, sociaux et moraux de ses membres ${ }^{37}$.

La preuve révèle qu'aujourd'hui, l'APCPNHQ représente entre $80 \%$ et $86 \%$ des cadres de premier niveau répartis dans l'ensemble des divisions et au sein du secteur corporatif d'HydroQuébec.

Au fil des ans, différentes ententes portant sur des conditions de travail ont été conclues entre les parties sans toutefois que la négociation ayant mené à leur terme puisse être qualifiée de la même nature que celle préconisée pour la conclusion d'une convention collective. D'ailleurs, la dernière entente (qui date de 2010) réfère à des « encadrements » de l'entreprise ou à des "règles de gestion » édictées par l'employeur qui n'ont aucunement fait partie des discussions entre les parties. La décision ne précise pas si tel était le cas dans les versions précédentes, mais il y a lieu de croire qu'il en était ainsi. Élément important soulevé par la juge Zaikoff, le terme «négociation » n’apparaît nulle part à cette entente. On y parle plutôt de « discussions » entre les parties.

La suite de la décision fait état longuement des différentes difficultés révélées par la preuve en ce qui a trait notamment à la relation entre les parties pour l'application de l'entente de travail et des mécanismes de communication qui y sont prévus. Selon les faits établis par la partie requérante, la dynamique relationnelle semble en être une où l'employeur se sert du canal de l'entente de travail pour faire part à ses cadres de premier niveau des décisions qu'il a prises à la suite des rencontres entre les parties.

\subsubsection{Chez la Société des casinos du Québec}

La Société des casinos du Québec est une filiale de la Société des loteries du Québec (connue sous le nom de Loto-Québec). Elle est responsable de la gestion des quatre casinos situés sur le territoire de la province. Cinq paliers de directions sont en place dans cet organisme pour le secteur des jeux : directeur général, directeur des jeux, deux chefs de services, chefs d'opération et les superviseurs des opérations, lesquels superviseurs forment ce qu'il est convenu d'appeler au présent débat les cadres de premier niveau. Le secteur des jeux comprend les tables de jeux, les machines à sous, le Keno (une sorte de bingo) et un salon de poker.

Le rôle des cadres de premier niveau (superviseur des opérations) consiste à superviser les croupiers aux tables de jeux qui, eux, sont des employés syndiqués. Le superviseur des opérations veille au bon déroulement des activités, plus particulièrement tout ce qui concerne le respect des règles du jeu, et il assume également la responsabilité du service à la clientèle. Entre deux et cinq croupiers sont sous la supervision de chacun. Comme mentionné à la décision, les cadres de premier niveau sont « les yeux et les oreilles de l'employeur sur le plancher ${ }^{38}$. Cette catégorie de poste est issue d'une restructuration organisationnelle effectuée en 2005 alors que des responsabilités accrues à l'égard de la supervision des croupiers ont été confiées aux anciens chefs de tables et, du coup, le palier de supervision qui se situait au-dessus d'eux a été aboli. Une décision du tribunal en 1995 avait clairement établi (dans le cadre d'une requête en accréditation) que les anciens chefs de tables avaient le statut de représentant de l'employeur, ce qui les excluait de l'unité de négociation des croupiers ${ }^{39}$.

À la suite d'une modification unilatérale par l'employeur des horaires de travail des chefs de tables en 1996, ceux-ci décident de former l'année suivante l'«Association des superviseurs de tables de jeux du Casino de Montréal » en vertu de la Loi sur les syndicats professionnels, laquelle représentait uniquement les chefs de tables. En 2001, cette association prendra le nouveau nom de l'« Association des cadres de la Société des casinos du Québec (ACSCQ) » en vue de représenter désormais tous 
les cadres de premier niveau. Un protocole d'entente interviendra ultérieurement avec l'employeur en vue d'officialiser le tout à l'établissement de Montréal ainsi qu'à celui de Lac-Leamy.

L'ACSCQ représente 250 superviseurs des opérations affectés aux tables de jeux de deux casinos tout comme un certain nombre d'autres superviseurs des opérations affectés à d'autres divisions pour lesquels cependant l'employeur refuse d'étendre le champ d'action de l'ACSCQ. Au moment du dépôt de la requête en accréditation, l'employeur reconnaît l'ACSCQ uniquement à titre de représentant des superviseurs des opérations aux tables de jeux. La requête déposée fait état d'une demande d'unité de négociation qui regrouperait également les cadres de premier niveau des autres divisions du secteur des jeux (machines à sous, Keno et salon de poker).

Malgré des demandes répétées de l'ACSCQ, l'employeur a toujours refusé d'élargir la reconnaissance de sa représentation à l'ensemble des superviseurs des opérations. Le principal argument pour justifier son refus est que l'ACSCQ ne représente pas la majorité des superviseurs des opérations dans ses autres secteurs des jeux. Selon l'employeur, la reconnaissance majoritaire doit être établie dans chacune des divisions (machines à sous, Keno et salon de poker) et non pas globalement.

Peu après sa formation en 1997, l'ACSCQ a adhéré à la Conférence nationale des cadres du Québec (CNCQ). Elle en est toujours membre au moment de l'audition. Au terme de deux rencontres de travail tenues en mars et septembre 2007 entre les représentants de la CNCQ et ceux du gouvernement du Québec, l'Association des cadres de la Société des casinos du Québec (ACSCQ) a demandé aux représentants du gouvernement d'être reconnue à titre de représentant des cadres de premier niveau, tous secteurs confondus et dans l'ensemble des casinos. Elle réclame de plus l'établissement d'un mécanisme de règlement des différends, entre autres, pour les cas de fin du lien d'emploi.

En ce qui concerne les conditions de travail, le protocole d'entente réfère à un manuel des employés édicté par l'employeur. En 2004, un document intitulé "Guide des conditions de travail » est annexé au "Manuel du personnel cadre du casino » où plusieurs demandes faites antérieure- ment par l'ACSCQ trouvent écho. Il est important toutefois de noter que ce document est l'œuvre unilatérale de l'employeur, l'ACSCQ n'ayant aucunement été consultée préalablement à son adoption. Une modification surviendra en 2012 où, encore là, l'association ne sera pas consultée. Les échelles salariales sont révisées annuellement au terme de ce document, tout comme les augmentations de salaire ainsi que les bonis (dont les règles d'octroi ont été modifiées unilatéralement par l'employeur autour de 2010). Aucune négociation entre les parties n'intervient sur ces sujets, la décision définitive revenant à l'employeur.

Comme cela semble être le cas pour l'Association professionnelle des cadres de premier niveau d'Hydro-Québec (APCPNHQ), l'Association des cadres de la Société des casinos du Québec est de façon générale informée par l'employeur de la détermination des conditions de travail à intervenir et aucune négociation n'est tenue en ce sens entre les parties, hormis toutefois l'établissement des horaires de travail pour lesquels les travaux d'un comité mixte établi en 2003 ont porté ses fruits. Depuis, même s'il y a eu reconduction desdits horaires, l'ACSCQ n'a pas été préalablement consultée, exception faite de quelques occasions où des modifications ont été apportées à la demande de l'association. Depuis 2013, il ne semble plus y avoir de collaboration entre les parties à ce chapitre alors que l'employeur a retiré unilatéralement un horaire de travail précédemment établi, d'où la plainte en vertu de l'article 59 C.t. déférée à l'arbitrage en août 2014.

\subsection{Les prétentions des parties}

De part et d'autre, l'argumentation prend ses assises sur les décisions de la Cour suprême du Canada en matière de droit à l'association. Il va de soi cependant que les parties ne partagent pas les mêmes points de vue quant à la portée de ces enseignements.

Principalement, les deux associations requérantes plaident que l'exclusion de leurs membres du cadre associatif prévu par le Code du travail entrave de manière substantielle ${ }^{40}$ leur droit à un véritable processus de négociation collective. Les éléments suivants sont soulevés pour étayer cette prétention : 
- Insuffisance de l'indépendance de l'association

- Impossibilité de négocier collectivement les conditions de travail

- Absence d'un mécanisme permettant d'établir un rapport de force entre les parties

- Absence de recours à un tribunal spécialisé pour sanctionner le manquement à l'obligation de négocier de bonne foi

- Non-existence du droit de grève ou d'un mécanisme permettant de régler les litiges

En définitive, on y retrouve là les éléments de ce qu'il est convenu d'appeler « la nouvelle trilogie du droit du travail » telle qu'établie par la Cour suprême du Canada lors du prononcé de trois décisions concomitantes en janvier $2015^{41}$.

En contrepartie, l'argument principal soumis par les deux employeurs repose sur la prétention qu'il faut distinguer entre le droit d'association et le droit à l'accréditation, lesquels ne sont pas directement reliés. Donc, pour eux, l'exclusion d'une catégorie de personnes de l'application des dispositions sur le droit d'association prévues au Code du travail n'est pas en soi une violation de la liberté d'association. Ce faisant, les deux associations requérantes devraient démontrer que cette exclusion rend à toutes fins utiles impossible la constitution d'une association et qu'il y a là « entrave substantielle » à l'exercice de la liberté d'association.

Quant à l'obligation de négociation de bonne foi, les employeurs allèguent que le Code civil du Québec (CCQ-1991) y pourvoit déjà et qu'en cas de manquement, un recours devant le tribunal de droit commun peut être exercé.

La Procureure générale du Québec étant interpellée par la nature de la demande (la contestation de la constitutionnalité d'une disposition d'une loi du Québec), elle a fait valoir certains arguments de droit d'ordre plus général. Par souci d'économie, nous n'en ferons pas état, sauf en ce qui concerne l'obligation de loyauté inhérente à la fonction de cadre en entreprise et l'élément de confidentialité qui en découle, lesquels justifieraient selon ses prétentions la constitutionnalité de la disposition contestée du Code du travail.

Revue Organisations \& Territoires • Volume 26 • N 1 1-2 2017

\section{La décision du Tribunal administratif du travail (TAT)}

\subsection{Les décisions antérieures de la Cour suprême du Canada}

La liberté d'association est un des grands droits que protègent autant la Charte canadienne que la Charte provinciale. Citant à cet effet la Cour suprême du Canada, la juge Zaikoff, d'entrée de jeu, situe la place qu'occupe ce droit sur l'échiquier constitutionnel :

(49) Le droit d'association ne représente pas un droit simplement dérivé des autres droits et libertés garantis par la Constitution. Au contraire, il constitue un droit distinct doté d'un contenu autre, un droit essentiel au développement et au maintien de la société civile dynamique sur laquelle repose notre démocratie $e^{42}$.

(Soulignement ajouté)

Par la suite, faisant état de l'évolution du droit d'association élaboré par la Cour suprême du Canada entre ce qu'il est convenu d'appeler « la trilogie de $1987 »^{43}$ et « la trilogie de $2015 »^{44}$, elle expose les préceptes établis par le plus haut tribunal au pays.

La trilogie de 1987 avait amené la Cour suprême à définir la portée de la liberté d'association selon les droits que possède chaque individu, sans qu'ils puissent être élargis du fait de l'association. Ainsi, le droit à l'association se voulait à l'époque purement à titre individuel, le droit de négocier collectivement tout comme le droit de faire la grève n'étant aucunement considérés (nonobstant la dissidence du juge en chef Dickson dans Renvoi relatif à l'Alberta où ce dernier était d'avis que certaines activités collectives pouvaient faire l'objet d'une protection par la Charte).

Par la suite, avec l'arrêt Dunmoret5 en 2001, la Cour suprême décida dans ce cas précis que le caractère vulnérable du groupe visé entraînait pour l'État une obligation d'intervention positive afin de permettre l'association en vue de négocier de façon plus efficace. Le tribunal en vient à la conclusion que l'exclusion des travailleurs du régime général d'accréditation viole la liberté d'association.

L'arrêt Health Services ${ }^{46}$ en 2007 permet finalement à la Cour suprême du Canada d'aborder plus spécifiquement le droit à la négociation collective. Elle conclut 
que l'alinéa 2d) de la Charte canadienne protège le droit des salariés de participer à un véritable processus de négociation collective de leurs conditions de travail. La cour précise toutefois que c'est le processus qui est protégé, et non le résultat. C'est à cette décision que le concept de «l'entrave substantielle » apparait; l'atteinte à la liberté de négociation doit, selon le tribunal, être substantielle. Ainsi, sera substantielle l'entrave qui affecte la réalisation des objectifs poursuivis et le processus lui-même. L'entrave doit donc avoir des incidences sur la capacité de négocier collectivement et toucher des sujets d'importance pour l'activité collective. La négociation collective de bonne foi impliquera alors un effort raisonnable pour arriver à un compromis acceptable.

S'ensuivit en 2011 l'arrêt Frasert7 où des travailleurs contestaient une loi ontarienne mettant en place un régime particulier pour les travailleurs agricoles. La cour détermine dans ce cas que la loi contestée ne viole pas l'alinéa $2 \mathrm{~d}$ ) de la Charte canadienne. Selon le tribunal, cette loi permettait de participer à un processus de négociation collective puisque les travailleurs pouvaient former une association, formuler des revendications collectives à l'employeur et les voir prises en compte. La contestation du syndicat fut jugée prématurée car il n'avait pas tenté d'utiliser le mécanisme prévu par la loi, soit le recours à un tribunal spécialisé créé pour régler les différends (lequel permettait de s'assurer du respect de l'obligation de négocier de bonne foi).

La trilogie de $2015^{48}$ permet en somme de cristalliser le changement d'approche entrepris à partir de la décision Dunmore (2001). Dans l'arrêt Association de la police montée de l'Ontario, la Cour suprême affirme que le droit à la négociation collective fait partie intégrante de la liberté d'association, laquelle est protégée par la Charte canadienne et n'est pas simplement un droit « dérivé » comme cela avait été qualifié à l'arrêt Fraser (2011). Le rapport de force dans les relations de travail entre employés et employeur est au cœur même de la liberté d'association. Il faut alors se demander si la mesure contestée perturbe l'équilibre des rapports de force que l'alinéa 2d) vise à établir de telle sorte que cela interfère de façon "substantielle » avec un processus véritable de négociation collective.

À l'arrêt Meredith, la Cour suprême du Canada juge que la loi contestée (une loi fédérale restreignant les augmentations salariales dans le secteur public) ne viole pas la liberté d'association des membres de la GRC, car elle vise tous les fonctionnaires de la province et cette loi n'interdit pas les consultations sur d'autres questions salariales.

Finalement, l'arrêt Saskatchewan concerne le droit de grève des employés du réseau de la santé qui se voit restreint par l'adoption d'une loi provinciale. La Cour suprême consacre ici le droit de grève au rang des droits constitutionnellement protégés par la liberté d'association prévue à l'alinéa 2d) de la Charte canadienne. Étant partie intégrante du droit de négocier collectivement, si celui-ci est limité d'une manière qui entrave «substantiellement» le processus de négociation collective, il doit être remplacé par un mécanisme de règlement des différends.

\subsection{Le régime législatif d'accréditation au Québec}

Le droit d'association déterminé à l'article 3 du Code $d u$ travail 49 s'inspire des principes de la loi « Wagner » aux États-Unis. Conséquemment, il est indéniable que le but de toute accréditation est la négociation collective. Au terme des dispositions de l'article $53^{50}$ du Code du travail, celle-ci doit « commencer et se poursuivre avec diligence et bonne foi ».

Le Code du travail favorise l'accréditation. Elle est la règle générale et les exclusions du droit à celle-ci doivent être vues de manière exceptionnelle. C'est donc dans ce contexte d'exception que la définition de « salarié » de l'article 1 du Code du travail prévoit nommément à son sous-paragraphe l) $1^{\circ}$ qu' « une personne qui, au jugement du tribunal est employée à titre de gérant, surintendant, contremaître ou représentant de l'employeur dans ses relations avec ses salariés » n'est pas comprise à la définition de «salarié».

Le concept de cadre en entreprise se veut assez vaste. On peut viser des personnes qui simplement exercent un pouvoir de surveillance et veillent à l'application des directives de l'employeur. À l'autre extrémité du spectre, les personnes qui participent à l'élaboration des stratégies et des orientations sont également couvertes par cette notion. Au fil des ans, la jurisprudence et la doctrine en sont venues à concevoir trois paliers de cadres : les cadres de premier niveau, les cadres intermédiaires et les cadres supérieurs. Le sousparagraphe 1 ll $1^{\circ}$ couvre les trois (3) niveaux de cadres, sans distinction. 


\subsection{L'exclusion du statut de cadre viole-t-elle la liberté d'association?}

À cette question le tribunal répond positivement.

De façon générale, l'objet de l'exclusion des cadres de la notion de «salarié » contenue au Code du travail vise à empêcher des représentants de l'employeur de se placer en conflit d'intérêts en devant négocier collectivement leurs conditions de travail.

Pour en arriver à pareil constat, il faut regarder chacun des aspects couverts par la liberté d'association telle que reconnue par la Cour suprême du Canada et déterminer si les effets causés par cette atteinte constituent une entrave substantielle au droit de négocier collectivement.

Dans la décision Association de la police montée de l'Ontario, la Cour suprême établit qu'un processus véritable de négociation nécessite le droit pour les employés de former une association indépendante de la direction et le droit d'y adhérer.

Après analyse, la juge Zaikoff en vient à la conclusion que la reconnaissance des deux associations requérantes par leurs employeurs s'effectue sur une base volontaire. Le caractère représentatif tout comme la détermination du groupe des employés visés relèvent de l'entière discrétion des employeurs. Dans les deux cas, s'il existe des désaccords sur les personnes pouvant être membres des associations (ce qui est le cas à la Société des casinos du Québec), seuls les employeurs décident de l'issue des litiges. L'ingérence ou l'entrave aux activités des associations peut survenir et aucune protection n'existe à cet effet. D'ailleurs, la Cour suprême a déterminé que ce dernier élément est de première importance pour l'atteinte de l'objectif de la négociation collective.

Quant à la capacité de négocier collectivement les conditions de travail, force est de constater qu'il y a déséquilibre du rapport de force entre les parties. Le tribunal constate l'existence de ce déséquilibre de la manière suivante :

(372) En l'espèce, les personnes en cause sont des cadres de premier niveau, dans une organisation qui comprend cinq paliers ou plus de gestion. Ils sont souvent issus eux-mêmes du groupe qu'ils supervisent. Tout en étant « les yeux et les oreilles de l'employeur sur le plancher », ils ne bénéficient pas de la relation privilégiée que peuvent entretenir les cadres de niveaux supérieurs avec l'entreprise. Ils ne participent pas aux orientations de l'entreprise. Ils ne jouent pas non plus de rôle stratégique dans les relations du travail : ils ne négocient pas les conventions collectives, ils en assurent l'application dans le quotidien des activités. En résumé, les cadres de premier niveau sont véritablement entre «l'arbre et l'écorce ».

(373) À cela s'ajoute qu'en l'espèce, les employeurs en cause, des sociétés d'État, sont des entités puissantes, possédant d'importants moyens, et étroitement liées à l'appareil gouvernemental, dont elles bénéficient du soutien. La complexité de leur structure et le nombre d'intervenants alourdissent aussi la tâche des associations ${ }^{51}$.

La liberté d'association protège le processus de négociation, mais pas l'atteinte d'un résultat. Ce processus de négociation ne doit pas se limiter à un simple exercice de façade. Un véritable dialogue doit se mettre en place en vue de conclure un contrat de travail acceptable. Au terme de l'analyse du Tribunal administratif du travail, il ne semble pas que tel soit le cas pour les deux associations requérantes. La preuve soumise démontre que plusieurs sujets fondamentaux ne sont tout simplement pas ouverts à la discussion entre les parties.

(385) Étant exclues du régime général prévu au Code et en l'absence d'un régime répondant aux exigences constitutionnelles, l'ACSCQ et l'APCPNHQ n'ont pas accès à un mécanisme permettant d'assurer le respect des obligations de négocier de bonne foi et avec diligence ${ }^{52}$.

Contrairement aux prétentions des employeurs, le recours à un tribunal de droit commun pour sanctionner l'obligation de négocier de bonne foi n'a aucune commune mesure avec ce qu'il en est dans le cas d'un recours introduit devant une instance spécialisée. L'accessibilité, la rapidité et l'expertise en relations de travail y sont définitivement manquantes.

Finalement, l'exclusion du régime général a pour effet de priver les cadres de premier niveau de pouvoir exercer le droit de grève. Moyen privilégié en vue d'assurer une participation véritable au processus de 
négociation collective, c'est par le rapport de force que le droit de grève établit entre les parties qu'il justifie son importance. Au terme de la décision de la Cour suprême à l'arrêt Saskatchewan, le législateur ne peut limiter ce droit d'une manière qui entrave substantiellement le processus de négocier collectivement. Dans le cas présent, il a tout simplement été supprimé sans être remplacé par un autre mécanisme, constituant du coup une entrave substantielle.

Les associations de cadres de premier niveau ont démontré qu'elles cherchent à exercer leur droit à un véritable processus de négociation collective. C'est leur incapacité à le faire en raison de leur exclusion du Code du travail et l'absence d'un régime législatif leur donnant accès au droit à la négociation collective qui constitue une entrave substantielle. Ce faisant, l'État a une responsabilité dans l'entrave substantielle au processus de négociation collective des deux associations requérantes. Le modèle de relations de travail préconisé pour ces deux associations de travailleurs n'établit pas l'équilibre essentiel à la tenue d'une véritable négociation collective.

De manière plus spécifique dans le cas HydroQuébec, le tribunal détermine que les éléments suivants sont manquants en vue de justifier l'exclusion au droit à la négociation collective :

- Les cadres de premier niveau demeurent souvent tenus à l'écart par la direction qui prend des décisions les affectant sans consulter leur association.

- Les mécanismes de consultation sont défaillants ou insuffisants, ce qui empêche un processus véritable de négociation sur des objets importants.

- L'entente de travail intervenue n'est pas respectée par l'employeur ou son interprétation est sujette à désaccord, sans mécanisme indépendant de résolution des différends.

\subsection{Cette violation se justifie-t-elle dans le cadre d'une société libre et démocratique?}

La dernière étape de l'analyse du tribunal consiste à déterminer si la violation à la liberté d'association telle qu'établie peut se justifier en vertu de l'article 1 de la Charte canadienne ou de l'article 9.1 de la Charte québécoise ${ }^{53}$.
Selon la décision de la Cour suprême du Canada dans l'affaire Association de la police montée de l'Ontario,

(139) [...] Cette procédure de justification exige que l'objectif de la mesure soit urgent et réel et que le moyen choisi pour l'atteindre soit proportionné à cet objectif, c.-à-d. qu'il possède un lien rationnel avec l'objet de la loi, qu'il porte le moins possible atteinte au droit garanti par l'al. 2 d) et qu'il soit proportionné sur le plan de ses effets (R. c. Oakes, [1986]1 RCS 103; Health Services, par 137-139) ${ }^{54}$.

Selon les employeurs et la Procureure générale du Québec, l'exclusion des cadres de premier niveau serait nécessaire afin de conserver l'unité de direction dans des relations de travail de type «Wagner» où les deux parties risquent éventuellement de confronter leurs intérêts. L'obligation de loyauté jumelée à la volonté de vouloir éviter les conflits d'intérêts justifierait alors leur exclusion au droit à la négociation collective. Cet argument n'est pas retenu par le tribunal qui ne voit aucune justification entre l'exclusion des cadres de premier niveau du droit à l'association et le modèle de relations de travail de type « Wagner».

Quant à l'obligation de loyauté, même si celle-ci incombe à tout salarié comme l'exigent les dispositions du Code civil du Québec portant sur le contrat de travail ${ }^{55}$, il n'y a rien selon le tribunal qui ferait en sorte que des cadres devraient être privés de leur droit à négocier collectivement sur cette base. Rien ne justifie un lien entre la privation à ce droit et l'atteinte de l'objectif de loyauté.

Bref, voir la syndicalisation comme un mode qui nuit nécessairement aux relations du travail est dépourvu de fondement aux dires du tribunal. D'ailleurs, la Cour suprême du Canada affirmait le contraire dans sa décision Association de la police montée de l'Ontario : « le respect de la liberté d'association peut même assurer plutôt que compromettre l'existence de bonnes relations et ainsi en renforcer la stabilité $»^{56}$.

À la lumière de l'ensemble de ces considérants, la conclusion du tribunal nous semble sans équivoque. Les trois paragraphes suivants résument bien l'ensemble des éléments pris en considération, lesquels paraissent en conformité des enseignements de la Cour suprême du Canada : 
(501) Le tribunal arrive à la conclusion que l'exclusion des cadres de la définition de salariés dans le contexte soumis porte atteinte à la liberté d'association garantie par l'alinéa $2 d$ ) de la Charte canadienne et par l'article 3 de la Charte québécoise et qu'elle n'est pas justifiée dans une société libre et démocratique.

[...]

(503) Ainsi, leur indépendance est incomplète, leur reconnaissance dépend entièrement de leur employeur respectif et il n'existe aucune protection contre l'ingérence. Cette exclusion entrave substantiellement leur capacité à négocier sur des objets importants. L'absence d'un mécanisme permettant de sanctionner l'obligation de négocier de bonne foi et la suppression du droit de grève, sans alternative, ne permettent pas (sic) rétablir le rapport de force entre les cadres de premier niveau et les employeurs, des sociétés d'État, ce qui constitue une entrave substantielle dans un tel contexte.

$[\ldots]$

(505) Cette atteinte n'est pas justifiée en regard de l'article 1 de la Charte canadienne et de l'article 9.1 de la Charte québécoise. Le gouvernement n'a pas démontré que l'objet de l'exclusion du statut de cadre du Code, soit la prévention des conflits d'intérêts et de l'ingérence de l'employeur auprès des autres employés, ainsi que le maintien d'un équilibre lors d'un conflit de travail, était réel et urgent. De plus, il n'y a pas de lien rationnel entre l'objet de la mesure et l'atteinte aux droits. Enfin, cette atteinte n'est pas minimale. Par conséquent, cette exclusion est inopposable à la partie demanderesse et ne peut empêcher l'examen de sa requête en accréditation ${ }^{57}$.

En conséquence, le tribunal administratif du travail déclare inopérant le sous-paragraphe $1 l$ ) $1^{\circ}$ du Code $d u$ travail dans le cadre de la requête en accréditation des associations de cadres de premier niveau au motif que cette disposition légale porte atteinte à la liberté d'association garantie par les Chartes. Conséquemment, le tribunal avise les parties qu'elles seront convoquées à une audience du Tribunal administratif du travail (TAT) en vue de disposer des requêtes en accréditation déposées.

Prenant soin de souligner que la Cour suprême du Canada a statué qu'un tribunal administratif ne peut prononcer une déclaration d'inconstitutionnalité générale $^{58}$, il peut toutefois déclarer l'invalidité de la disposition attaquée aux fins de l'affaire dont il est saisi; ce qui fut fait dans le cas présent. Encore là, la conclusion se veut en lien avec les enseignements de la Cour suprême du Canada.

\section{La suite légale des événements}

Au terme de cette décision, le Tribunal administratif du travail (TAT) refusa la demande de suspendre de ses effets la décision rendue afin de permettre au législateur de prendre les mesures qu'il pourrait juger utiles quant au choix d'un régime approprié pour l'accréditation des cadres de premier niveau ${ }^{59}$.

Le 6 janvier 2017, les deux employeurs se sont pourvus en contrôle judiciaire de la décision du Tribunal administratif du travail (TAT) et les deux dossiers ont été réunis pour la suite des procédures. C'est dans ce contexte que la juge Chantale Châtelain de la Cour supérieure a ordonné le sursis des procédures. Faisant état des critères applicables pour l'octroi d'une telle demande de sursis, le tribunal y est allé des commentaires suivants, lesquels nous semblent à-propos pour la suite des événements :

(26) L'invalidité ou l'inapplicabilité constitutionnelle de l'exclusion de tous ou d'une certaine catégorie de cadres de la définition de salariés au Code du travail est une question complexe qui ne semble pas avoir été tranchée à ce jour. Selon la SCQ et HQ, question sur laquelle le tribunal ne se prononce pas, la réponse à cette question requiert notamment de se pencher sur l'équilibre des rapports de forces (sic) entre employeurs et salariés que le Code du travail a cherché à établir et si ce rapport de force est rompu par l'inclusion de cadres, quel que soit leur catégorie, dans la définition de salariés.

$[\ldots]$

(35) [...] les Décisions du TAT, en prononçant l'inapplicabilité constitutionnelle de l'exclusion des cadres dans la définition de salariés au Code du travail met (sic) en place un tout nouveau paradigme dans le domaine des relations de travail.

(36) Or, il est acquis que le Code du travail est une loi d'intérêt public qui vise le bien commun et le fait que la SCQ et HQ y soient soumises malgré la volonté du législateur, et ce, avant que la question constitutionnelle ne soit 
tranchée dans le cadre des pourvois en contrôle judiciaire constitue, de l'avis du Tribunal, un préjudice irréparable ${ }^{60}$.

Dans l'état actuel du dossier, celui-ci en est au stade de pourvoi en contrôle judiciaire auprès de la Cour supérieure. Les mémoires des employeurs ont été déposés au tribunal en juillet et août dernier de sorte qu'une décision est possible dans un avenir plus ou moins rapproché. Il va de soi que, eu égard à l'envergure et l'importance de la problématique soumise, des décisions de la Cour d'appel du Québec et, possiblement de la Cour suprême du Canada, s'ensuivront. À tout le moins, elles seront vivement souhaitées!

\section{Conclusion}

Au terme de la décision rendue par le TAT, doit-on conclure que désormais tous les cadres de premier niveau de quelque entreprise que ce soit auront droit à l'accréditation en vertu du Code du travail? Nous croyons qu'il y a loin de la coupe aux lèvres pour affirmer pareille chose.

Dans un premier temps, même si la décision nous semble en lien avec les enseignements de la Cour suprême du Canada, il faudra attendre la confirmation des tribunaux judiciaires au regard des principes établis aux deux décisions concernées. Il est important de remarquer que, tel que précédemment affirmé, le Tribunal administratif du travail (TAT) n'a pas décrété l'inconstitutionnalité du sous-paragraphe 1 ll) $1^{\circ} \mathrm{du}$ Code du travail puisque cette instance n'a pas le pouvoir de le faire. Ce n'est que la non-application de cette disposition du Code du travail qui a été décidée dans les deux cas soumis. Il sera intéressant de voir si les tribunaux judiciaires suivront la voie tracée par le TAT et déclareront l'inconstitutionnalité du sous-paragraphe 1 l) $1^{\circ} \mathrm{du}$ Code du travail; eux ont ce pouvoir.

Le fait que les deux associations requérantes font partie d'organisations paragouvernementales avec plusieurs niveaux hiérarchiques particularise la décision du TAT. Il ne faut toutefois pas exclure d'emblée tout organisme du secteur privé quant à une éventuelle application de ces principes de droit. Le cas d'entreprises commerciales d'envergure comportant différents niveaux hiérarchiques risquerait d'amener à la même conclusion dans la mesure où les éléments soulevés par la Cour suprême du Canada à ses trois décisions de 2015 s'y retrouvent.
Dans l'état actuel des choses, des interrogations subsistent au terme de la décision rendue. Ainsi, il existe une distinction entre le fait d'avoir le droit de négocier collectivement ses conditions de travail et celui d'avoir accès à un régime précis de relations de travail (l'accréditation en vertu du Code du travai). C'est d'ailleurs là le principal argument soulevé par les employeurs dans leur opposition à la demande des associations et la décision nous a semblé peu loquace sur ce sujet. Y aura-t-il lieu pour les tribunaux judiciaires de préciser ce point?

S'il s'avère un jour que cette décision serve de précédent pour l'établissement du droit à l'accréditation des cadres de premier niveau, certains sujets devront faire l'objet de clarification. Les cadres qui n'ont pas de réels pouvoirs de gérance deviendront-ils automatiquement « syndicables » au terme de cette nouvelle approche du droit à l'association? Qu'adviendra-t-il, par exemple, des demandes d'accréditation en semblable contexte lorsque l'unité de négociation antérieurement reconnue chez un employeur vise « tous les salariés au sens du Code du travail »? Pourra-t-on alors conclure automatiquement que le groupe des cadres de premier niveau forme une unité de négociation plus appropriée qui justifierait son accréditation indépendante?

À court terme, s'il s'avère que la décision du TAT soit confirmée, celle-ci aura des répercussions majeures en cas de conflit de travail. Les cadres de premier niveau devenant des salariés au sens du Code du travail, leurs services ne pourront plus être requis en cas de grève ou lock-out. En raison du nombre de personnes concernées, il pourrait devenir, en pareil contexte, difficile pour les deux employeurs concernés par l'actuel débat de maintenir les opérations en cas de conflit de travail. Il en sera probablement de même pour toute organisation où les cadres de premier niveau obtiendront l'accréditation. Des batailles juridiques pour délimiter la frontière entre le cadre intermédiaire et le cadre de premier niveau seront à prévoir puisque l'enjeu sera de première importance pour le maintien des opérations advenant un conflit de travail ${ }^{61}$.

Doit-on conclure que toute exclusion des cadres du statut de salarié, telle qu'elle figure actuellement au Code du travail, risque de disparaitre? Nous ne le croyons pas. Le cadre supérieur devra toujours maintenir une obligation de loyauté et éviter les conflits d'intérêts inhérents à sa fonction, d'où la justification 
de son exclusion. En sera-t-il de même dans le cas du cadre intermédiaire?

S’il advenait que les tribunaux judiciaires confirment la règle établie en ce cas précis par le Tribunal administratif du travail, nous croyons qu'une intervention du législateur sera inévitable en vue de délimiter le champ d'action de ce qu'il conviendra d'appeler un «cadre de premier niveau ». Dans cet ordre d'idées, le Code du travail devra encadrer le droit à la négociation collective pour ces derniers à la lumière des préceptes établis par la Cour suprême du Canada. La suite des événements sera à suivre!

\section{NOTES}

1 Association professionnelle des cadres de premier niveau d'Hydro-Québec (APCPNHQ) et Hydro-Québec, 2016 QCTAT 6871; Association des cadres de la Société des casinos du Québec et Société des casinos du Québec Inc., 2016 QCTAT 6870.

2 Instance aujourd'hui remplacée par le Tribunal administratif du travail (TAT).

3 1. Dans le présent code, à moins que le contexte ne s'y oppose, les termes suivants signifient : $[\ldots]$ l) «salarié » : une personne qui travaille pour un employeur moyennant rémunération, cependant ce mot ne comprend pas :

$1^{\circ}$ une personne qui, au jugement du Tribunal, est employée à titre de gérant, surintendant, contremaitre ou représentant de l'employeur dans ses relations avec ses salariés;

42010 QCCRT 0187.

52012 QCCS 112.

6 Ibid., pages 8 et 10 .

7 Une décision antérieure d'un commissaire du travail visant des employés occupant les mêmes fonctions que les requérants chez ce même employeur, décision confirmée par le Tribunal du travail en 1995, allait en ce sens : AZ-95147098 (T.T. 1995-09-21).

8 R. c. Conway, (2010) 1 RCS 765.

92014 QCCA 603; cité en page 6.

10 118. La Commission peut notamment:

$[\ldots]$

$4^{\circ}$ décider de toute question de droit ou de fait nécessaire à l'exercice de sa compétence;

N.B. L'article 118 CT a été abrogé lors de la mise en vigueur de la Loi instituant le tribunal administratif du travail (LTAT) le $1{ }^{\mathrm{er}}$ janvier 2016. C'est dorénavant l'article 9 LTAT qui couvre ce sujet.

11 2. Chacun a les libertés fondamentales suivantes:

$[\ldots]$ d) liberté d'association.

1. La Charte canadienne des droits et libertés garantit les droits et libertés qui y sont énoncés. Ils ne peuvent être restreints que par une règle de droit, dans des limites qui soient raisonnables et dont la justification puisse se démontrer dans le cadre d'une société libre et démocratique.

3. Toute personne est titulaire des libertés fondamentales telles la liberté de conscience, la liberté de religion, la liberté d'opinion, la liberté d'expression, la liberté de réunion pacifique et la liberté d'association.

9.1. Les libertés et droits fondamentaux s'exercent dans le respect des valeurs démocratiques, de l'ordre public et du bienêtre général des citoyens du Québec.

La loi peut, à cet égard, en fixer la portée et en aménager l'exercice.

12 59. À compter du dépôt d'une requête en accréditation et tant que le droit au lock-out ou à la grève n'est pas exercé ou qu'une sentence arbitrale n'est pas intervenue, un employeur ne doit pas modifier les conditions de travail de ses salariés sans le consentement écrit de chaque association requérante et, le cas échéant, de l'association accréditée.

100.10. Une mésentente relative au maintien des conditions de travail prévu à l'article 59 ou à l'article 93.5, doit être déférée à l'arbitrage par l'association de salariés intéressée comme s'il s'agissait d'un grief.

132015 QCCS 4645.

14 Ibid., page 3.

152015 QCCA 2032. 
16 Ibid., page 3.

172017 QCCA 877.

18 Ibid., pages 12 et 13 .

19 Ibid., pages 18, 25, 27 et 28.

20 12. Aucun employeur, ni aucune personne agissant pour un employeur ou une association d'employeurs, ne cherchera d'aucune manière à dominer, entraver ou financer la formation ou les activités d'une association de salariés, ni à y participer.

21 Société de développement de la Baie-James et al. c. Chef Kanatewat et al., (1975), CA, 169.

222015 QCCRT 0342, p. 9.

23 Les audiences sur la question de la constitutionnalité de l'exclusion du statut de cadre ont débuté en mars 2015 pour se terminer en juillet 2016.

242016 QCTAT 6871, p. 2

25 Les limites prévues aux articles 1 de la Charte canadienne et 9.1 de la Charte québécoise; (note 11).

26 Convention $\left(n^{\circ} 87\right)$ sur la liberté syndicale et la protection du droit syndical, 1948.

Convention ( $n^{\circ}$ 98) sur le droit d'organisation et de négociation collective, 1949.

Pacte international relatif aux droits économiques, sociaux et culturels (ratifié par le Canada en 1976).

Pacte international relatif aux droits civils et politiques (également ratifié par le Canada en 1976).

27 Art. 3(1) [...] Sont exclus du champ d'application de la présente définition les personnes occupant un poste de direction ou un poste de confiance comportant l'accès à des renseignements confidentiels en matière de relations du travail.

28 Le Code canadien du travail s'applique aux entreprises œuvrant dans des secteurs d'activités relevant de la juridiction du gouvernement fédéral aux termes de la loi constitutionnelle.

29 S.Q. 1944 , c. 30

2. Dans la présente loi et son application, à moins que le contexte ne s'y oppose, les termes suivants ont le sens qui leur est donné ci-après :

a) « salarié » signifie : tout apprenti, manœuvre ou ouvrier non spécialisé, ouvrier qualifié ou compagnon, artisan, commis ou employé qui travaille individuellement, en équipe ou en société; cependant, ce mot ne comprend pas :

$1^{\circ}$ les personnes employées à titre de gérant, surintendant, contremaître ou représentant de l'employeur dans ses relations avec ses salariés;

30 Le dernier paragraphe de l'article 21 du Code du travail de 1970 se lisait comme suit :

(...) Les associations qui étaient reconnues par la Commission bydroélectrique du Québec ou la Ville de Montréal le 2 août 1969 pour représenter des groupes de personnes comprenant en totalité ou en partie des gérants, surintendants, contremaitres ou représentants de leur employeur dans ses relations avec ses salariés et qui, à cette date ou dans l'année précédant cette date, étaient à leur égard parties signataires à une entente collective de travail, sont à compter du 17 juillet 1970 des associations accréditées à leur égard comme si l'accréditation avait été accordée par un commissaire-enquêteur.

S. R. 1964 , c. 141 , a. 20; 1965 (1re sess.), c. 50, a. 2; 1969, c. 47, a. 9; 1969, c. 48, a. 9; 1970, c. 33, a. 1.

312016 QCTAT 6871; page 16.

32 Laquelle regroupe des associations de cadres municipaux et des réseaux de la santé et scolaires ainsi que les trois associations de cadres qui s'étaient jointes à la demande à l'Organisation internationale du travail (OIT) tel que mentionné au texte.

33 Rapport du Comité sur la liberté syndicale, n³ 335, novembre 2004; cité en page 17, 2016 QCTAT 6871.

34 Ibid. ; cité en page 19, 2016 QCTAT 6871.

35 Rapport du Comité sur la liberté syndicale, n³ 362, novembre 2011; cité en page 20, 2016 QCTAT 6871.

36 Cité à 2016, QCTAT; page 25.

37 6. Les syndicats professionnels ont exclusivement pour objet l'étude, la défense et le développement des intérêts économiques, sociaux et moraux de leurs membres.

382016 QCTAT 6870; page 25.

39 500-28-000102-95; 21 septembre 1995.

40 La Cour suprême du Canada a reconnu à la décision Health Services (Health Services and Support - Facilities Subsector Bargaining Assn. c. Colombie-Britannique [2007] 2 RCS 391.) que seule «l'entrave substantielle » sera considérée comme étant un obstacle à la liberté d'association. Sera substantielle selon le tribunal l'entrave qui affecte la réalisation des objectifs poursuivis et le processus lui-même. 
41 Saskatchewan Federation of Labour c. Saskatchewan [2015] 1 RCS 245; Association de la police montée de l'Ontario c. Canada (Procureur général) [2015] 1 RCS 3; Meredith c. Canada (Procureur général) [2015] 1 RCS 125.

42 Association de la police montée de l'Ontario c. Canada (Procureur général), [2015] 1 RCS 3; cité en page 51, 2016 QCTAT 6871.

43 En 1987, trois jugements en droit du travail ont été rendus par la Cour suprême du Canada : Renvoi relatif à la Public Service Employee Relations Act (Alb.) [1987] 1 RCS 313; AFPC c. Canada [1987]1 RCS 424; SDGMR c. Saskatchewan [1987] 1 RCS 460.

44 Note 41.

45 Dunmore c. Ontario (Procureur général) [2001] 3 RCS 1016.

46 Health Services and Support-Facilities Subsector Bargaining Assn. c. Colombie-Britannique [2007] 2 RCS 391.

47 Ontario (Procureur général) c. Fraser [2011] 2 RCS 3.

48 Note 41.

49 3. Tout salarié a droit d'appartenir à une association de salariés de son choix et de participer à la formation de cette association, à ses activités et à son administration.

50 53. La phase des négociations commence à compter du moment où l'avis visé à l'article 52 a été reçu par son destinataire ou est réputé avoir été reçu suivant l'article 52.2. Les négociations doivent commencer et se poursuivre avec diligence et bonne foi.

512016 QCTAT 6871; page 75.

52 Ibid., page 77

53 Note 11.

54 Note 41, cité à 2016 QCTAT 6871, page 91.

55 2088. Le salarié, outre qu’il est tenu d'exécuter son travail avec prudence et diligence, doit agir avec loyauté et honnêteté et ne pas faire usage de l'information à caractère confidentiel qu'il obtient dans l'exécution ou à l'occasion de son travail.

56 Note 41, cité à 2016 QCTAT 6871, page 97.

57 2016, QCTAT 6871, page 101.

58 Cuddy Chicks Limited c. Ontario (Commission des relations de travail), [1991] 2 RCS 17; cité à 2016 QCTAT 6871, page 98.

59 Tel que cela fut demandé par la partie intimée.

602017 QCCS 2525, pages 6-7.

61 Dans la mesure où le cadre intermédiaire n'aura pas droit à l'accréditation. 